\title{
EFFECT OF COLLAR AND BEVEL ANGLE IN MIXING ENHANCEMENT OF MACH 1.76 JET EXITING FROM A CONVERGENT-DIVERGENT NOZZLE
}

\author{
Bholu Kumar ${ }^{1}$, Suresh Kant Verma ${ }^{1}$, Shantanu Srivastava ${ }^{2 *}$ \\ ${ }^{1}$ Department of Mechanical Engineering, National Institute of Technology Patna, India \\ ${ }^{2}$ Department of Mechanical Engineering, Motilal Nehru National Institute of Technology Allahabad, India
}

\begin{abstract}
:
The present work focuses on the effect of nozzle collar exit inclination on the mixing characteristics of Mach 1.76 jet in both design and off-design conditions using commercial software package ANSYS Fluent. In order to maintain the uniform Mach number at the nozzle exit, a collar is inserted at the nozzle exit and collar exit is varied with bevel angle of $30^{\circ}, 45^{\circ}$ and $60^{\circ}$ to see the effect of bevel collar on the jet mixing. Thus, four model namely C30 ( $30^{\circ}$ bevel), C45 ( $45^{\circ}$ bevel), C60 $\left(60^{\circ}\right.$ bevel) and collar with zero bevel angle (UJ) are constructed for investigation. From the obtained results, it is reported that the bevel collars efficiently reduce the jet core as much as $76 \%$, indicating enhanced jet mixing and found effective in both design and off-design condition of the jet. The $\mathrm{C} 60$ jet shows highest jet mixing followed by C45 jet, C30 jet and UJ. Thus, increase in bevel angle led to increase in mixing enhancement of the jet. Due to asymmetry in collar, the upward and downward shift of the jet core are seen with variation in NPR for the controlled jets (C30, C45 and C60).
\end{abstract}

\section{ARTICLE HISTORY}

Received: 26.12.2021.

Accepted: 16.03.2021.

Available: 31.03.2021.

\section{KEYWORDS}

Supersonic jet, bevel collar, mixing, shock, SST k- $\omega$

\section{INTRODUCTION}

The mixing of the jet with surrounding mass of the ambient fluid is the major point of concern, as better mixing is favourable both from aero acoustics and aerodynamic points of view. For example, rapid the mixing between air and fuel in the combustion chamber led to enhanced mixing efficiency of the combustion cycle. The infrared signatures at the nozzle exit also reduces due to the enhanced mixing with the ambient fluid. Like these, the other applications of jet control also include noise suppression, metal deposition, thrust vector control, gas dynamic lasers etc. [1]. The study of supersonic axisymmetric jet issuing from the convergent-divergent nozzle such as circular nozzle, rectangular nozzle etc., has been investigated by many researchers in the recent past [2-6]. However, passive control technique such as alteration in nozzle exit cross-sections are shown excellent mixing enhancement of the jet with surrounding fluids [7-10]. Mixing enhancement of supersonic jets becomes extremely important after ample research has conclusively shown that compressibility effects reduce the growth rate of mixing layers [11-12]. Several engineering applications such as high-speed airbreathing engines (SCRamjet engines), supersonic ejectors, and aero acoustics noise of the jet exhaust are entirely dependent on the mixing characteristics at supersonic speed. Gutmark et al. [13] reviewed a wide range of supersonic mixing enhancement techniques, and described the effectiveness of passive techniques that use geometrical modification at the nozzle exit. Large streamwise vortices generated by such passively controlled 
nozzle was shown to have increased mixing and entrainment rate. Such convoluted nozzle generating streamwise vortices were particularly useful for applications in supersonic ejectors [10, 14] and for improvement in supersonic combustion [15]. However, large stagnation pressure loss was also reported because of complex shock structures generates from such nozzles [14]. Rao et al. [16] performed the comparative studies on nozzles of complex geometry at supersonic speed, Mach 1.8; the nozzles under considerations were a beveled nozzle, a nozzle with six chevrons and a six lobed Elliptic Sharp Tipped Shallow (ESTS) nozzle, and compared the results with reference nozzle of conical shape. The jet from bevel nozzle has its mixing characteristics modified due to the prevailing azimuthal asymmetry. The study of jets from beveled nozzles was first reported by Wlezien and Kibens [17]. The work involved investigation of flow field and noise-generation characteristics of jets from non-axisymmetric nozzles at supersonic pressure ratios. However, constant diameter tubes were used as non-axisymmetric nozzles, it was found that, the jet deflects are caused from the axis of the nozzle. One of the significant findings is that, these jets likely exhibit increased mixing because of the augmented shear-layer surface area. One of the earliest studies in supersonic jet noise reduction using nozzle trailing edge modification was conducted by Norum [18], when it was demonstrated that asymmetric jet nozzles could lead to significant reduction in jet screech amplitude levels. Rice and Raman [19] conducted studies on supersonic rectangular bevelled jets and observed significant increases in high frequency jet noise, even though peak mixing noise was reduced. Viswanathan [21] and Viswanathan Czeth [22] reported that bevelled nozzles lead to significant noise reduction along azimuthal directions below the longer nozzle lips. Furthermore, Power et al. [23] studied the acoustic properties of military style supersonic bevelled nozzle jets and noticed that a noise reduction of 3-4 decibel $(\mathrm{dB})$ could be achieved along the peak emission direction aligned with the longer nozzle lip region. Wu and New [24] investigated the effect of bevel angle on noise and shock patterns of the jet at supersonic speed of Mach 1.5. They found that, the pattern of shock in the jet core changes from diamond shape to triangular and then triangular to rectangular with changes in bevel angle and nozzle pressure ratio (NPR).
So far, it has been reported from the past literature that, most of the studies on the jet focused on the aero acoustics such as noise control. The mixing characteristics of jet from bevelled nozzle is remain outstanding. Thus, the present work focuses on the numerical investigation of the mixing characteristics of Mach 1.76 jet issuing from collar inserted at the nozzle exit with bevel angle of $30^{\circ}, 45^{\circ}$ and $60^{\circ}$ and without bevel angle. This study covers both design and off-design conditions of the jet with nozzle pressure ratio variation of 4.5 to 7.5 with step size of one. The results are also compared with flat collar (zero bevel angle) in order to see the effect of bevel exit inclination on jet mixing.

\section{METHODOLOGY AND PROCEDURES}

The two-dimensional numerical studies are conducted and the results obtained are compared with the work of Wu and New [24]. It is found that the results obtained are in good agreement with the work of $\mathrm{Wu}$ and New. Thus, two-dimensional CAD model of the convergent-divergent plain nozzle is constructed in the design modeler of ANSYS workbench 19.1. Assuming unit length normal to the plane of the paper, the design Mach number of the jet is found to be 1.76 with throat equivalent diameter of $13 \mathrm{~mm}$ and nozzle exit equivalent diameter of $18.15 \mathrm{~mm}$. The Area-Mach number relation is used to design the nozzle for Mach 1.76 jet [25]. The convergent angle of $30^{\circ}$ and divergent angle of $7^{0}$ is used in design of require convergentdivergent nozzle. A collar of uniform diameter, equal to equivalent diameter (D) of nozzle exit is extended about two times of equivalent diameter (D) of nozzle exit to get the uniform Mach number at the nozzle exit irrespective of bevel angle at the collar end. The nozzle with above collar arrangement is then enclosed by the rectangular domain of size 10D $\times 30 \mathrm{D}$. The schematic diagram is shown in Fig. 1. Thus, four distinct models are constructed using the design modeler of ANSYS workbench. The first model consists of collar with no exit inclination and thus designated as uncontrolled jet (UJ). However, the second, third, and fourth model consists of collar with bevel angle of $30^{\circ}, 45^{\circ}$, and $60^{\circ}$ respectively at the collar exit. Thus, the second model with $30^{\circ}$ of bevel angle at collar end is designated as C30 jet. The third and fourth models are designated as C45 jet and C60 jet respectively. 


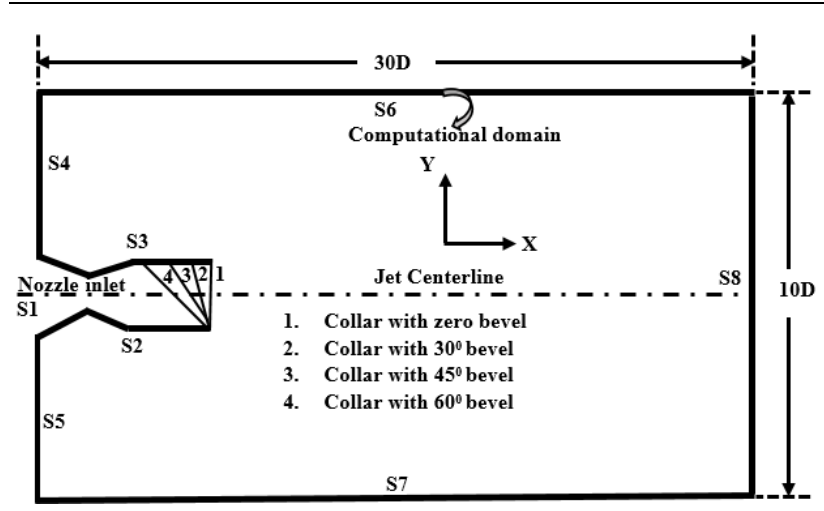

Fig. 1. Schematic diagram of computational domain including nozzles with collars

\subsection{Mesh Generation}

Each of the model is then imported into the ICEM module of ANSYS workbench for the mesh generation. Due to the interface between the nozzle and the domain, the entire domain is split into required number of rectangular subdomains to generate quad structured grids. The computational grids of one of the models (UJ) is shown in Fig. 2, and the grids for all other models are generated in similar way as that of UJ model. The grids near the nozzle and jet boundary are comparatively more refined in order to capture the effect of entrainment and turbulence on the mixing of the jet.

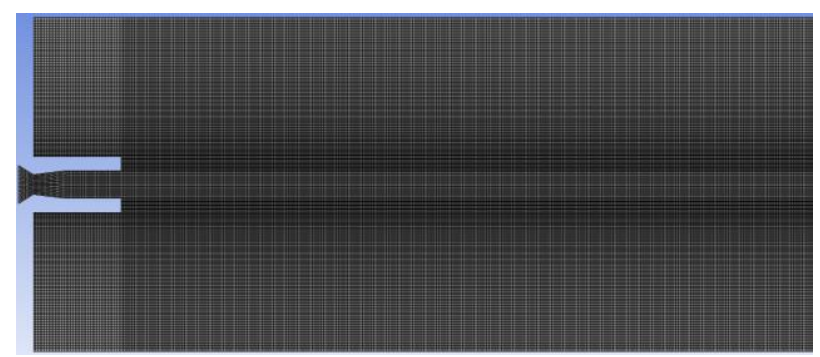

Fig. 2. Computation grid of the first model (UJ)

Three different numbers of grids are generated for all of the four computational models to check the mesh (or grid) independence of the results with grid size. The grid independence test (GIT) is mainly conducted to check at what and above grid size the results of the simulation become unchanged. The results of the first (UJ), second (C30), third (C45) and fourth (C60) models are found independent of grid size at 285150 cells, 361654 cells, 361654 cells, and 367470 cells respectively. The present the GIT, the local jet Mach number $\left(\mathrm{M}_{\mathrm{J}}\right)$ is non-dimensionalized by the correctly expanded Mach number (Mc) and plotted along non-dimensionalized transverse direction at the non-dimensional axis distance of $X / D=0$, as shown in Fig. 3 .

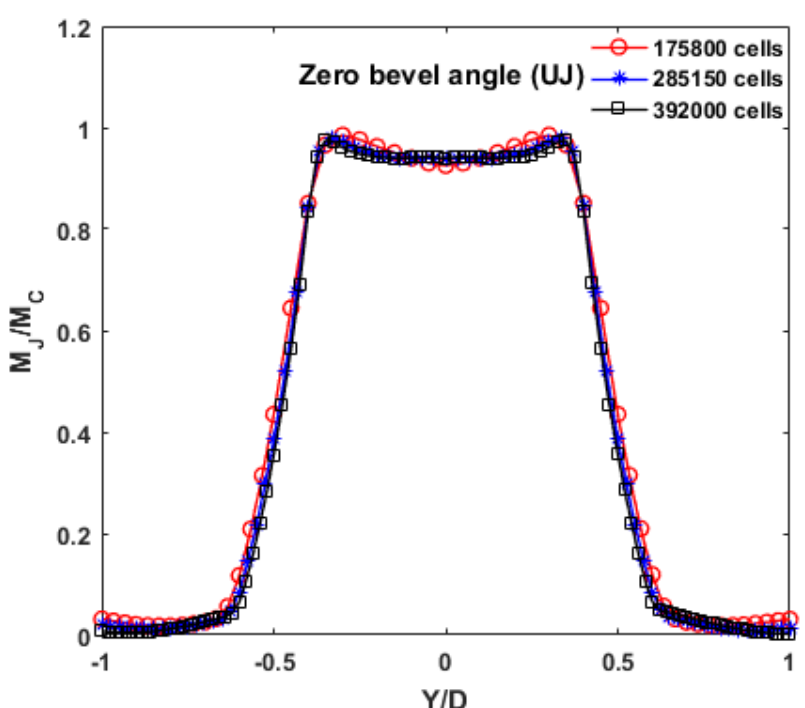

(a)

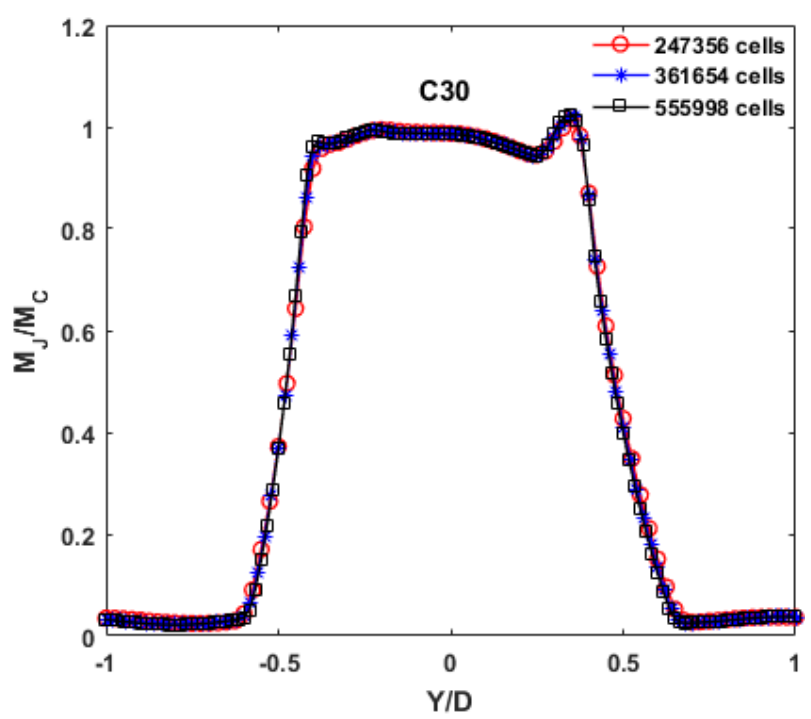

(b)

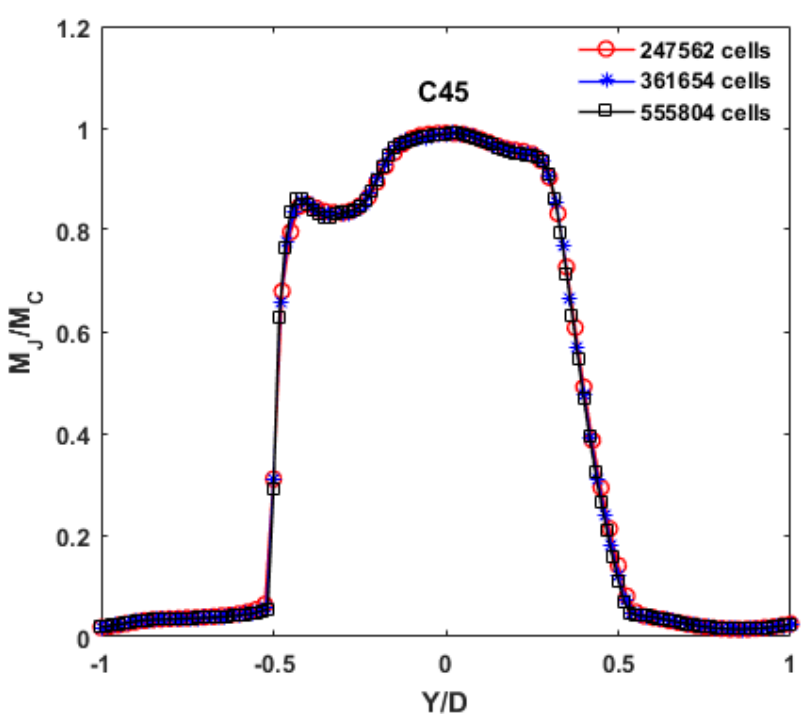

(c) 


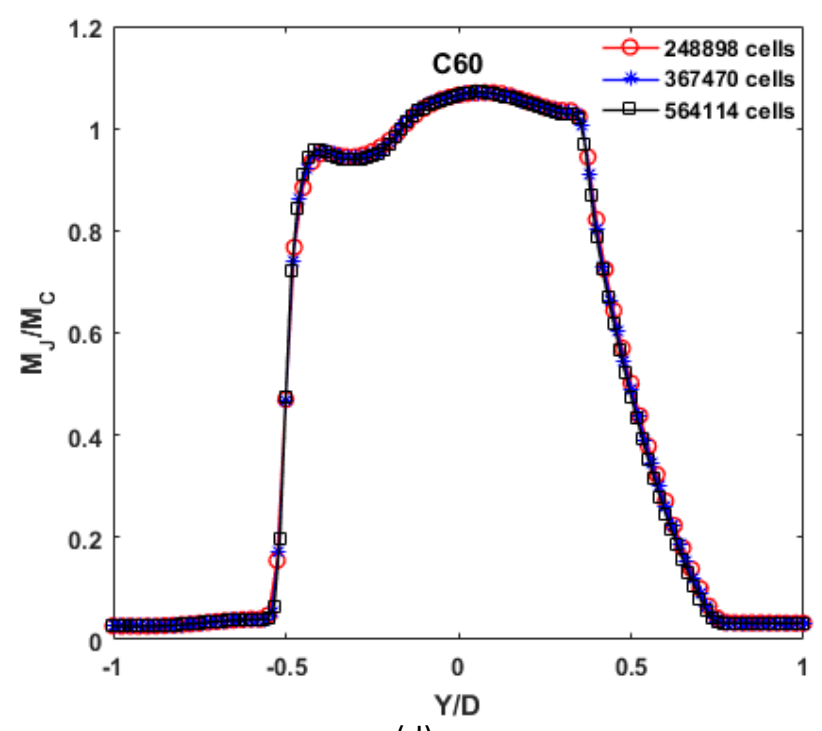

(d)

Fig. 3. Grid independence test for (a) Uncontrolled jet (UJ), (b) C30 model, (c) C45 model, and (d) C60 model

\subsection{Numerical Procedures}

The Fluent solver of the ANSYS workbench is used to get the numerical solutions. The Fluent solver uses the finite volume approach such that each of the control volumes (grids) discretized into simultaneous sets of algebraic equations from the differential form of the governing equations and the subsidiary equations. Fluent solver then uses numerical methods techniques such as Gauss-seidel, Runge-kutta etc. to get the results in numeric form. The governing equations applied for the solution of the computational modes are as follow.

\subsection{Governing Equations}

The jet flow for this study involves the following governing equations.

- Continuity Equation

$$
\frac{\partial u_{i}}{\partial x_{j}}=0
$$

- Momentum Equation

$$
\begin{aligned}
\rho \frac{\partial\left(U_{i} U_{j}\right)}{\partial x_{j}}= & -\frac{\partial P}{\partial x_{i}}+ \\
& \frac{\partial}{\partial x_{i}}\left[\mu\left(\frac{\partial U_{i}}{\partial x_{j}}+\frac{\partial U_{j}}{\partial x_{i}}\right)-\rho \overline{U_{\imath} U_{J}}\right]
\end{aligned}
$$

The above governing equations with transport equations for turbulent kinetic energy $(k)$ and turbulent dissipation $(\omega)$ are uses to get the simultaneous sets of algebraic equations by applying suitable pressure-velocity coupling scheme, applied on each control volumes (grids). The sets algebraic equation obtained from each of the control volume is further solve with the numerical methods such as Runge-Kutta, GaussSeidel etc., to get the results in numeric forms.

\subsection{Turbulence Model}

The SST $k$ - $\omega$ turbulence model is adopted for the numerical simulation. The SST $k-\omega$ turbulence model is a hybrid turbulence model that includes the $k-\varepsilon$ and $k-\omega$ turbulence model [26]. The $k-\varepsilon$ turbulence model well captures the turbulence far from the wall; however, the $k-\omega$ turbulence model captures the turbulence near the wall. Hence, SST k$\omega$ turbulence model captures the turbulence in both the scenario. Thus, due to this robust quality, the SST k- $\omega$ turbulence model is frequently used by many researchers in the recent past [27-29].

- SST k-w Turbulence Model

$$
\begin{aligned}
& \frac{\partial(k)}{\partial t}+<u_{j}>\frac{\partial k}{\partial x_{j}}=\frac{\partial}{\partial x_{j}}\left[\left(v+\frac{v_{t}}{\sigma_{k}}\right) \frac{\partial k}{\partial x_{j}}\right]-\beta^{*} k \omega_{k}+ \\
& v_{t} S^{2} \\
& \frac{\partial \omega}{\partial t}+<u_{j}>\frac{\partial \omega}{\partial x_{j}}=\frac{\partial}{\partial x_{j}}\left[\left(v+\frac{v_{t}}{\sigma_{\omega}}\right) \frac{\partial \omega}{\partial x_{j}}\right]-\gamma S^{2}- \\
& \beta f_{\beta} \omega^{2}+2 \sigma_{\omega 2}\left(1-F_{1}\right) \frac{1}{\omega}
\end{aligned}
$$

The terms $\sigma_{k}, \omega_{k}, \gamma$, and $\beta$ in equations ( 3 ) and (4) are correlated with a coefficient $\varphi$ which is expressed as follow.

$\emptyset=F_{1} \emptyset_{1}+\left(1-F_{1}\right) \emptyset_{2}$

Now, the coefficient $\varphi_{1}$ and $\varphi_{2}$ as given in equation (5) adjust their value so that to capture the turbulence of flow in both near the wall and far from the wall by using a function $F_{1}$. For capturing turbulence near the wall $F_{1}$ adjust their value to 1 and thus turbulence capture by the $k-\omega$ model. However, for capturing turbulence far from the wall the function adjusts their value to 0 and thus enable the $k-\varepsilon$ model. Furthermore, in order to limit the turbulent viscosity in the region of adverse pressure gradient, Bradshaw [30] proposed the following hypothesis.

$v_{t}=\frac{a_{1} k}{\max \left(a_{1} \omega ; F_{2} \bar{\Omega}\right)}$

$\bar{\Omega}=\sqrt{2 \Omega: \Omega} \quad$ avec 
$\Omega_{i j}=\frac{1}{2}\left(\frac{\partial<u_{i}>}{\partial x_{j}}-\frac{\partial<u_{j}>}{\partial x_{i}}\right)$

The constant $a_{1}$ expressed in equation (6) is called Bradshaw constant and its numeric value found to be about 0.31 .

\subsection{Boundary Conditions}

The boundaries of the computational domain as shown in Fig. 1, is denoted by S1 to S8, where S1 represents nozzle inlet. The pressure intel boundary condition is applied at intel S1. Whereas, wall boundary condition with standard wall function is applied at the nozzle wall, denoted by S2 and S3. In addition to nozzle intel $\mathrm{S} 1$, pressure intel boundary condition is also given at the far-field of the computational model, denoted by S4, S5, S6 and S7. The outlet of the computational domain, denoted by $\mathrm{S} 8$ is specified by the pressure out boundary condition.

\subsection{Numerical Simulation}

The jet flow through entire computational domain is simulated using the steady-state pressure-based solver using Fluent 19.1. Assuming air is compressible, an ideal gas law with Sutherland viscosity formulation is set as properties of air. The pseudo transient method with coupled pressurevelocity coupling is set for the simulation. The pressure and convective term of the flow are discretized by second-order and second-order upwind scheme. Furthermore, full-multigrid initialization is run to get smooth and faster convergence of the solution. The convergence criteria of $1 \mathrm{e}-04$ is set.

\section{RESULTS AND DISCUSSION}

It has been established in literature [25] that, whenever subsonic jet (or sonic jet) expands in divergent portion of a typical convergent-divergent nozzle, it develops well defined flow zones outside the nozzle due the formation of shear layer. These flow zones are namely, potential core, characteristic decay and fully developed zones. Potential core is the region where jet centreline velocity remains constant once exiting the nozzle; thus, no mixing take place in this zone. After the potential core region, the jet centreline velocity start decaying due to the momentum exchange with the surrounding fluids which is termed as characteristics decay zone. Beyond the characteristics decay zone, the jet profile becomes self-similay in appearance and the region is known as fully-developed or self-similar region of the jet. However, whenever dealing with the supersonic jets, the potential core of the jet is characterized by wave-dominated region from the nozzle exit. The potential core of the supersonic jet is strongly dependent upon the stagnation pressure at nozzle exit and Mach number. The present study pay attention on length of potential core mainly as shorter will the potential core, higher will be the jet mixing [6]. The physics of jet mixing is nothing but role of vortex dynamics. Once jet issues from the nozzle exit into the quiescent medium of surrounding fluids, the vortical structures are formed and the large-scale vortices engulf the huge amount of surround mass into the jet filed and the process is known as entrainment. The vortices carrying fluid masses break down in smaller and smaller eddies and finally dissipate the energy as travel in downstream, and thus mixing between fluids having different momentum take place. To quantify the extent of jet mixing the Mach number in function of axial distance is presented and will discuss in ongoing sections. To report the results in the ongoing sections, it is important to note that the Mach number along the jet centreline is plotted in function of non-dimensional axial distance $(X / D)$, where $X / D=0$ represent the collar exit.

\subsection{Computational Validation}

The present investigation at design condition ( $M$ $=1.76, \mathrm{NPR}=5.5)$ is compared with the results of Wu and New [24] at design condition (M = 1.5, NPR = 3.4). The comparison is shown in Fig. 4. The core decay patterns are almost similar and the Mach levels of present study is higher due to the higher design value. At design conditions it is well known that flow exiting from the nozzle would have straight symmetrical flow about the jet centreline irrespective of geometrical conditions at same design Mach number. Thus, the results of present study and Wu \& New study at respective design conditions are very close irrespective of bevel angles. It is obvious from the Fig. 4 that, matching the design conditions of present study and study of Wu and New, the comparative plots of would come very closer to each other. Thus, the computational validation of present study is reasonable enough to carry out the further work and to report the new findings. 


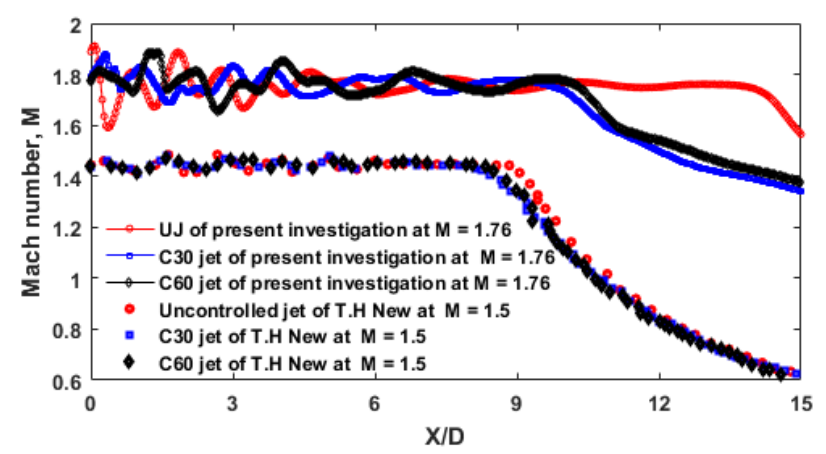

Fig. 4. Validation of present investigation with computational work of $\mathrm{Wu}$ and New [24]

\subsection{Centreline velocity decay}

Fig. 5, shows the Mach number (or velocity) decay of the jet along its centreline, where it is found to be highly over-expanded with an overexpansion level of about 18\%. Due to overexpanded condition of the jet, an adverse pressure gradient occur at the collar exit and pressure at the collar exit drop below the ambient pressure of the environment. However, in order to reach the equilibrium of the flow, the pressure of collar exit need to reach the level of ambient pressure and due to this reason oblique shock is formed at the edge of the collar exit which enhance the collar exit pressure and subsequently drop the axial velocity of the jet just near the collar exit. It is interesting to note that, due to oblique shock occur at the edge of collar exit the Mach number of exit should decrease but in reality, the Mach number of the jet first increases due to relaxation provided by the large space of environment and thereafter the Mach number decrease due to the formation of oblique shock. That is why the Mach number of the jet for all the models (UJ, C30, C45, and C60) considered has increased at the collar exit, Fig. 5 . The increase in Mach number is due to the expansion fan provided by the large space of environment at the collar exit. The oblique shocks of opposite family coming from the edge of collar exit meet at the jet centreline (called crossover point), becomes stronger in strength and leads to huge drop in Mach number downstream of the collar exit.

Fig. 5, shows drop in Mach number for C45 and C60 jets to the distance $0.25 \mathrm{D}$ and $0.5 \mathrm{D}$ downstream of the collar exit respectively. However, UJ and C30 jets decelerates to 1D and $1.25 \mathrm{D}$ once leaving the collar exit. The oblique shock from the crossover point reach to the jet boundary reflected as expansion wave and flow gets accelerated and cycle is repeated till flow becomes low subsonic. Due to cycle of compression and expansion waves sinusoidal profiles occur in the potential core of the jet.

At NPR 4.5, four number of shocks are seen for UJ and the shocks present in the jet core appeared stronger in strength and longer in length when compared to controlled jets (C30, C45, and $\mathrm{C} 60$ jets). However, only three shocks are seen for the control jets (C30, C45, and C60) and the shock strength and shock length decrease with increase in bevel angle, thus $\mathrm{C} 60$ jet shows faster jet core decay followed by C45, C30 and UJ. The core reduction of $68 \%, 64 \%$, and $36 \%$ respectively is reported for $\mathrm{C} 60, \mathrm{C} 45$, and C30 jets as compared to UJ.

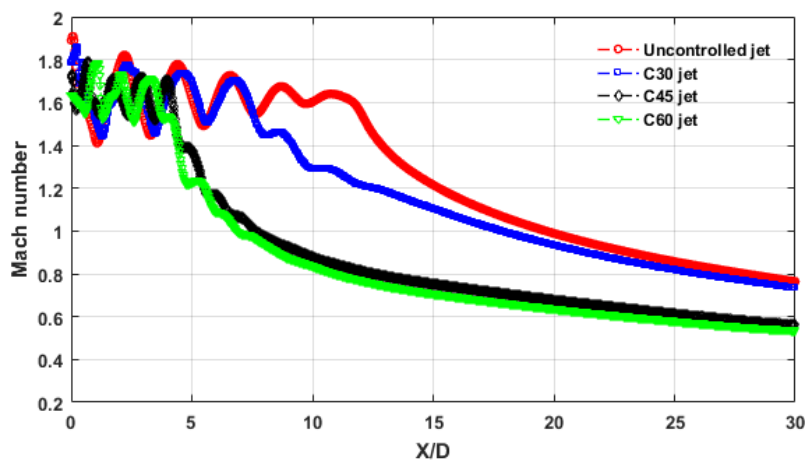

Fig. 5. Centreline velocity decay of over-expanded jet at NPR 4.5

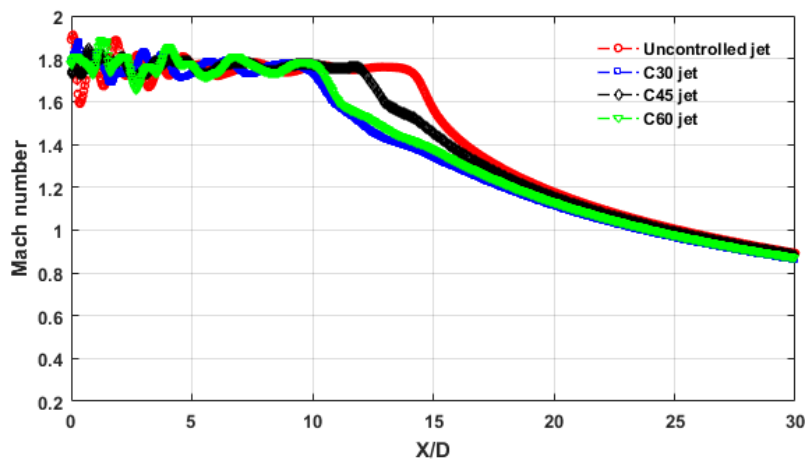

Fig. 6. Centreline velocity decay of correctly-expanded jet at NPR 5.5

At NPR 5.5 the jet is correctly expanded, Fig. 6, i.e., the collar exit pressure matches to pressure of outside environment and neither adverse pressure gradient nor favourable pressure gradient occur at the collar exit. But, in reality flow expands from the exit due to large space of the environment. Since supersonic flow is wave dominated flow and any change in its state or direction led to formation of shocks. That is why even at zero pressure gradient at the collar exit the flow first expands and then compress and repeat the cycle as similar to overexpanded and under-expanded jet. The only difference in correctly expanded jet is that their compression and expansion strength are weak as compared to over-expanded and under-expanded 
jets. So, due to state of correctly expanded condition (design condition) all of the models (UJ, C30, C45, and C60) show almost same flow physics with minor wavy nature. At design condition, the jet exiting from symmetrical nozzles (circular, square) exhibit symmetrical core from the jet centreline. However, for unsymmetrical nozzle (here bevel nozzle) perfect symmetry is not possible due to the different shock deflection angle at the exit. That is why the core decay of above models occurs randomly and due to this, the core decay of C30 and C60 jets start earlier as compared to C45 jet and UJ. Due to the design condition, leisurely core reduction is anticipated even using jet control (C30, $\mathrm{C} 45$, and $\mathrm{C} 60$ jets). The core reduction of $36 \%, 28 \%$, and $14 \%$ are respectively reported for $\mathrm{C} 30, \mathrm{C} 60$, and C45 jets as compared to UJ.

At NPR 6.5 as shown in Fig. 7, the jet at collar exit becomes under-expanded with an under-expansion level of about $18 \%$ and in this case the favourable pressure gradient occurs at the collar exit. Due to this the jet exiting from collar into free environment expand more as that of over-expanded and correctly expanded jet. That is why it is clearly seen from Fig. 7 and Fig. 8 that core of uncontrolled jet (UJ) has expanded more as that of controlled jets (C30, C45, and C60 jets). But, due to provision of collar exit inclination the jet core length is significantly reduced.

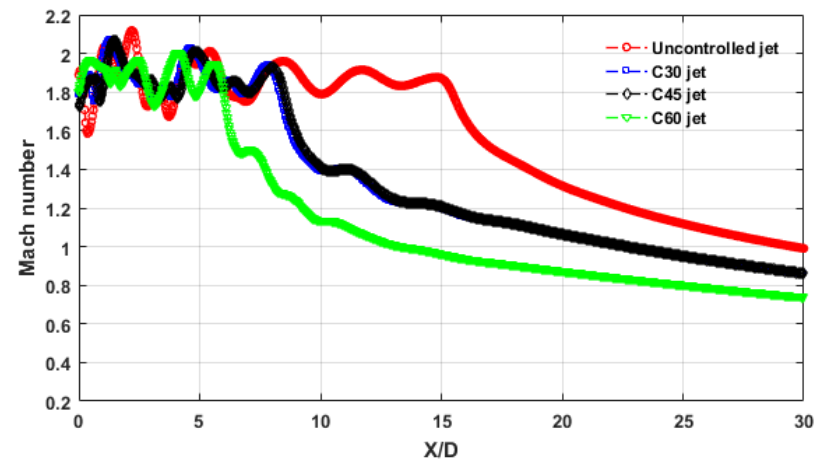

Fig. 7. Centreline velocity decay of under-expanded jet at NPR 6.5

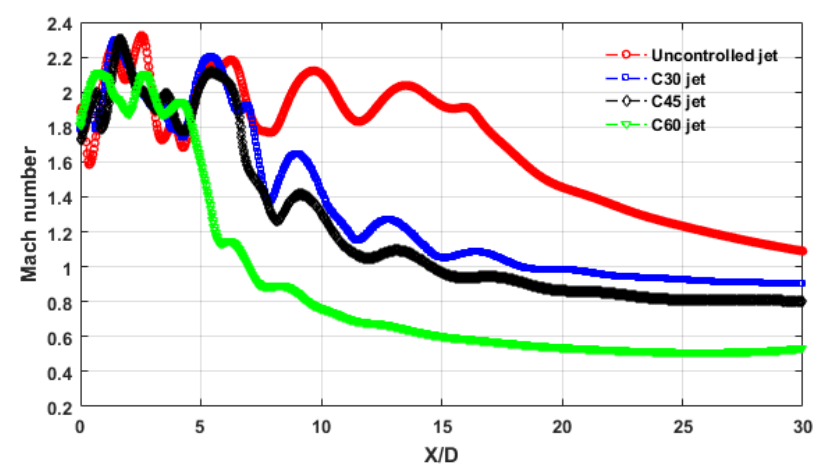

Fig. 8. Centreline velocity decay of under-expanded jet at NPR 7.5
It is also interesting to note that, the $\mathrm{C} 30$ and $\mathrm{C} 45$ jets give the same extent of jet mixing (Fig. 7 and Fig. 8) in the under-expanded conditions. However, highest jet mixing is seen from the $\mathrm{C} 60$ jet in underexpanded conditions (Fig. 7 and Fig. 8). The jet core reduction of about $47 \%$ is reported for $\mathrm{C} 30$ and $\mathrm{C} 45$ jets as compared to UJ at NPR 6.5. However, the core reduction of about $63 \%$ is reported by $\mathrm{C} 60$ jets as compared to UJ at NPR 6.5.

At NPR 7.5, Fig. 8, the jet is highly over-expanded with an over-expansion level of about $27 \%$ and due to this higher favourable pressure gradient occur at the collar exit. The shocks present in the core of UJ becomes longer and stronger in strength due to additional expansion provided by the large space of environment and also due to favourable pressure gradient. However, due to bevel collar inclination at the exit, the jet core has significantly reduced which led to enhanced jet mixing. The jet core reduction of about $64 \%$ is reported by $\mathrm{C} 30$ and $\mathrm{C} 45$ jets as that of UJ. However, the highest core reduction of about $76 \%$ is reported by $\mathrm{C} 60$ jet as that of UJ at NPR 7.5

\subsection{Qualitative visualization of shock present in the core of jet}

The Mach contours along the jet centreline has been plotted to qualitatively visualize the effect of shock present in the core of supersonic jet. For uncontrolled jet (UJ), Fig. 9, it is nice to see that the jet exiting from collar is found to be symmetrical about jet centreline. The shock length and strength increase with increase in NPR (NPR 4.5 to NPR 7.5).

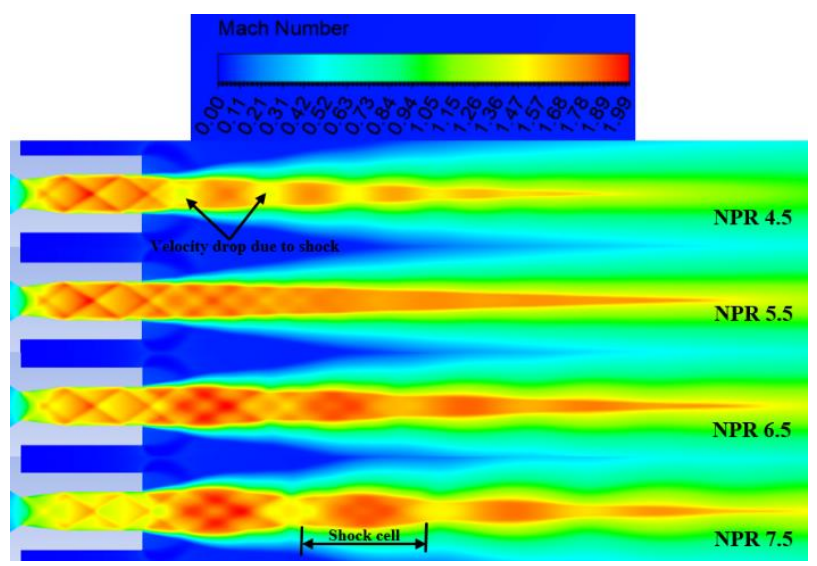

Fig. 9. Mach contour of uncontrolled jet (UJ)

The total four number of shocks is seen for uncontrolled jet, but the shock length and strength is found to be different with different NPR. At design condition (NPR 5.5), the shock present in jet core is of mild strength and due to this, the shocks are not appearing nicely however, the jet core 
decay plot (Fig. 6) authenticate the presence of four number of shock in the core of UJ. Furthermore, it is nice to see that the core of jet in off-design condition shows mixture of shock-cell structures that are; triangular, diamond, and rectangular. However, beautiful diamond shocks are only seen from the core of jet at design conditions and, these shocks are very closer, and thus it led to least pressure drop which in turn less thrust loss. That is why the jet engines and rocket engines perform the best at the design condition.

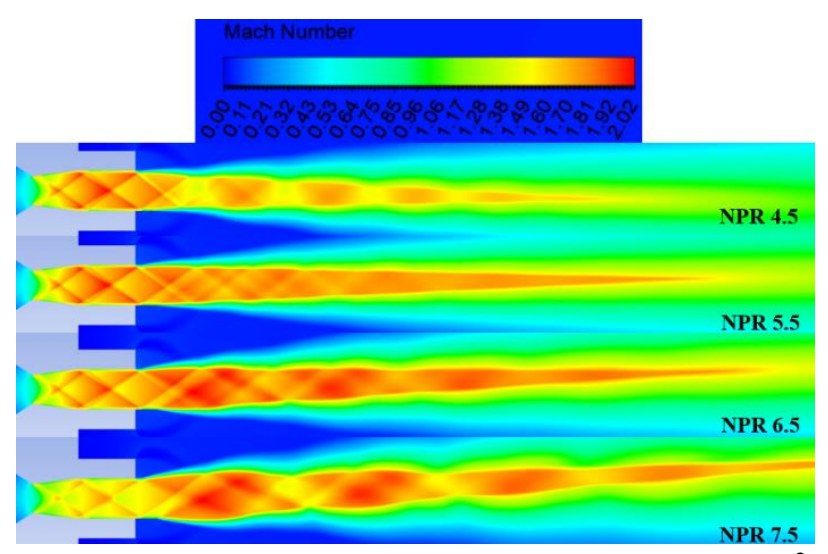

Fig. 10. Mach contour of jet exiting from collar with $30^{\circ}$ bevel angle

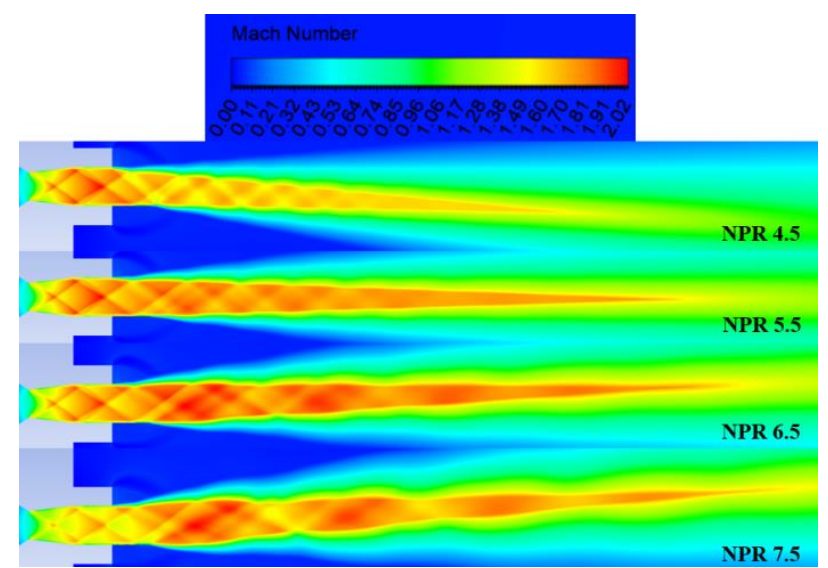

Fig. 11. Mach contour of jet exiting from collar with $45^{\circ}$ bevel angle

The scenario of jet exiting from collar with bevel angle is totally different as the shock coming from the top edge and bottom edge (lip) of the nozzle collar exit meet each other at jet centreline with different shock deflection angle. It is established in literature [31] that whenever shock of opposite family meets each other with different shock deflection angle there will be shift from the jet centreline with change in NPR and bevel angle. Furthermore, the shock deflection angle changes with NPR too and the entire jet core shifted in the direction of shock of higher defection angle. Due to this reason the core of $\mathrm{C} 30$ (shown in Fig. 10) jet is almost symmetrical from the geometrical centreline of the nozzle ( $x$-axis) at NPR 4.5 with total four number of shock present in its core. The symmetry in jet core is due to the almost same shock deflection angle of the shocks exiting from bevel collar at NPR 4.5. But as NPR increases the shock deflection angle of shock coming from top edge of the collar seems to be higher and thus the core of C30 jet start moving upward with increase in NPR. The irregular kind of shock cell is due to the interaction of shock of opposite family with different shock deflection angle itself.

Again, the core of $\mathrm{C} 45$ and $\mathrm{C} 60$ jets are different than UJ and C30 jets as shown in Fig. 11 and Fig. 12. Here the cores of $\mathrm{C} 45$ and $\mathrm{C} 60$ jets shifted downward form geometrical axis of the nozzle at NPR 4.5 due to the higher shock deflection angle of collar lip and as NPR increases the shock deflection angle of the shock coming from top edge of collar increases. Due to this reason the core of $\mathrm{C} 45$ and C60 jets moves upwards which becomes almost symmetrical about geometrical centreline of the jet at NPR 5.5, and above the geometrical centreline of the jet at NPR 6.5 and NPR 7.5.

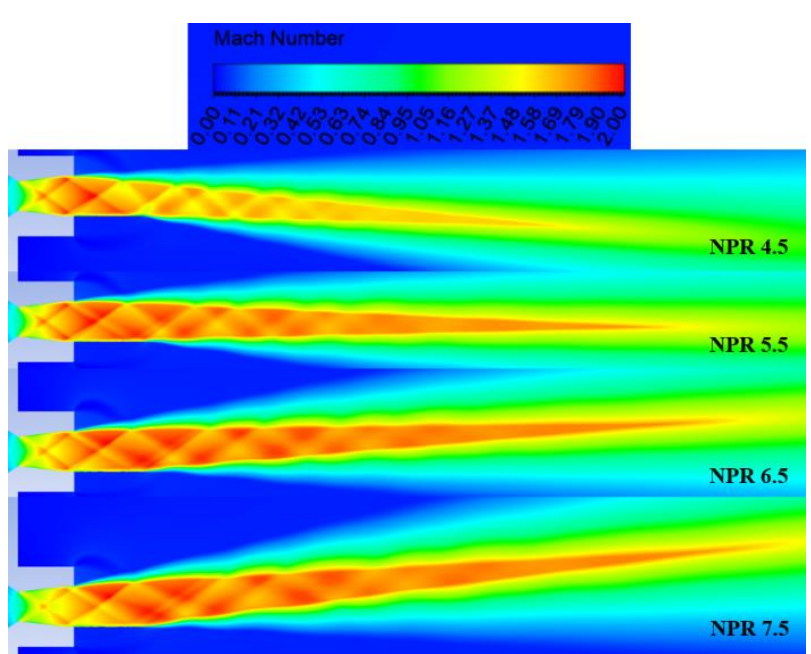

Fig. 12. Mach contour of jet exiting from collar with $60^{\circ}$ bevel angle

It is also interesting to see that the jet exiting from bevel collar evolve its cross-section in transverse direction of the jet and thus led to higher jet spread due to axis switching. It is also interesting to note that at design condition (NPR 5.5) all of the models of jet come symmetrical to geometrical centreline of the nozzle and appears visible diamond shock into the core of the jets. However, at off design conditions (over-expansion and underexpansion) the core of the unsymmetrical jets (C30, $\mathrm{C45}$, and (60) deviates from the geometrical centreline of the nozzle and shock appeared in its core are of mixed type (diamond and triangular, and rectangular). 


\section{CONCLUSION}

The investigations of mixing characteristics of Mach 1.76 supersonic jet exiting into from bevel collar is novelty in this study. The following findings are concluded from the study.

- The jet control in order to enhance the jet mixing is found effective in both design (correct expansion) and off-design conditions (overexpansion and under-expansion).

- The C60 jet shows highest core reduction followed by C45 jet, C30 jet, and UJ in off-design condition. The highest core reduction of about $76 \%$ is reported by $\mathrm{C} 60$ jet as compared to UJ. Thus, higher collar exit inclination led to higher jet mixing.

- At design condition, all of the computational models (UJ, C30, C45, and C60) shows clean and symmetrical flow about jet centreline with presence of diamond shock in the core of jet.

- At off-design conditions, the mixed shocks (diamond, rectangular and triangular) are seen in the core of jet.

- The asymmetry in collar exit causes axis switching of the jet which led to rapid mixing in the transverse direction and thus caused higher jet mixing. Thus, bevel collar is found beneficial in order to enhanced the jet mixing.

\section{FUTURE SCOPE}

The present investigations were mainly focused on the mixing enhancement of jet using collar and different bevel angle at collar end. The mixing enhancement is beneficial both from aerodynamics (base heat reduction in launch vehicle) and aero acoustics (noise suppression) point of view. However, when efficiency and performance of jet engine is considered then the thrust loss of engine is major concern which is not addressed in this study as study was focused on the mixing enhancement only. It was interesting to report that with increase in bevel angle the mixing gets improved but, there might be possibility of higher pressure drop inside the nozzle collar due higher bevel angle which leads higher thrust loss. Therefore, it would be interesting to see that with increase in bevel angle how much engine thrust is getting affected. This study would be helpful in deciding optimized bevel angle for optimum jet mixing and also for optimum performance of the jet engine.

\section{REFERENCES}

[1] E. Gutmark, F. F. Grinstein, Flow control with noncircular jets. Annual review of fluid mechanics, 31 (1), 1999: 239-272.

https://doi.org/10.1146/annurev.fluid.31.1.239

[2] E. Rathakrishnan, Experimental studies on the limiting tab. AIAA Journal, 47(10), 2009: 24752485.

https://doi.org/10.2514/1.43790

[3] S. Srivastava, M. Kaushik, Supersonic square jet mixing in presence of cross-wire at nozzle exit, Am. J. Fluid Dyn, 5, 2015: 19-23.

https://doi.org/10.5923/s.ajfd.201501.03

[4] E. Rathakrishnan, S. Srivastava, Performance of Corrugated Limiting Tab in Presence of Sharp Corners. In 49th AIAA/ASME/SAE/ASEE Joint Propulsion Conference, 2013, p.3975.

[5] D. Lohia, B. Kumar, S. Srivastava, H. Paliwal, Numerical simulation of supersonic overexpanded jet from 2-D convergentdivergent nozzle. International Journal of Integrated Engineering, 10(8), 2018: 195-201.

https://doi.org/10.30880/ijie.2018.10.08.029

[6] B. Kumar, S. Srivastava, Modelling 2-D Supersonic Jet from a Convergent-Divergent Nozzle using k- $\varepsilon$ Realizable Turbulence Model. Journal of Physics: Conference Series, 1240 (1), IOP Publishing, 2019.

[7] R. Srisha MV, G. Jagadeesh, Novel supersonic nozzles for mixing enhancement in supersonic ejectors. Applied Thermal Engineering, 71 (1), 2014: 62-71.

https://doi.org/10.1016/j.applthermaleng.2014.06. $\underline{025}$

[8] K. Fanshi, Y. Jin, T. Setoguchi, H. Dong Kim, Numerical analysis of Chevron nozzle effects on performance of the supersonic ejectordiffuser system. Journal of Thermal Science, 22 (5), 2013: 459-466.

http://dx.doi.org/10.1007/s11630-013-0648-4

[9] S. AJ, J. Kurian, V. Sriramulu, Experimental study on mixing enhancement by petal nozzle in supersonic flow. Journal of Propulsion and Power, 12 (1), 1996: 165-169.

https://doi.org/10.2514/3.24006

[10] T. Tillman, R. Paterson, W. Presz Jr, Supersonic nozzle mixer ejector. Journal of Propulsion and Power, 8 (2), 1992: 513-519.

https://doi.org/10.2514/6.1989-2925 
[11] D. Papamoschou, R. Anatol, The compressible turbulent shear layer: an experimental study. Journal of fluid Mechanics, 197 1988: 453-477.

\section{https://doi.org/10.1017/S0022112088003325}

[12] N. Clemens, M. Mungal. Large-scale structure and entrainment in the supersonic mixing layer. Journal of Fluid Mechanics, 284, 1995: 171-216.

https://doi.org/10.1017/S0022112095000310

[13] E. Gutmark, K. Schadow, K.H. Yu, Mixing enhancement in supersonic free shear flows. Annual Review of Fluid Mechanics, 27, 1995: 375-417.

https://doi.org/10.1146/annurev.fl.27.010195.002 $\underline{111}$

[14] A. Narayanan, K. Damodaran, Supersonicejector characteristics using a petal nozzle. Journal of Propulsion and Power, 10 (5), 1994: 742-744.

https://doi.org/10.2514/3.23788

[15] A. Srikrishnan, J. Kurian, V. Sriramulu. An experimental investigation of thermal mixing and combustion in supersonic flows. Combustion and flame, 107 (4), 1996: 464-474.

https://doi.org/10.1016/S0010-2180(96)00084-3

[16] M. Rao, A. Shingo, and S. Tsutomu, Comparative studies on supersonic free jets from nozzles of complex geometry. Applied Thermal Engineering, 99, 2016: 599-612.

https://doi.org/10.1016/j.applthermaleng.2016.01. $\underline{104}$

[17] R. Wlezien, V. Kibens, Influence of nozzle asymmetry on supersonic jets. AIAA journal, 26 (1), 1988: 27-33.

https://doi.org/10.2514/3.9846

[18] T. Norum, Screech suppression in supersonic jets. AIAA Journal, 21 (2), 1983: 235-240.

https://doi.org/10.2514/3.8059

[19] E. Rice, G. Raman, Supersonic Jets from Bevelled Rectangular Nozzles. NASA Technical Memorandum, 1993: 10640.

[20] G. Raman, Screech tones from rectangular jets with spanwise oblique shock-cell structures. In $34^{\text {th }}$ Aerospace Sciences Meeting and Exhibit, 1996.
[21] K. Viswanathan, Nozzle shaping for reduction of jet noise from single jet. AIAA Journal, 43 (5), 2005: 1008-1022.

https://doi.org/10.2514/1.11331

[22] K. Viswanathan, Krishna, M. Czech. Adaptation of the beveled nozzle for high-speed jet noise reduction. AIAA journal, 49 (5), 2011: 932-944.

https://doi.org/10.2514/1.J050409

[23] R. Powers, M. Dennis, Acoustics measurements of scale models of military style supersonic beveled nozzle jets with interior corrugations. $18^{\text {th }}$ AIAA/CEAS Aeroacoustics Conference, 2012.

https://doi.org/10.2514/6.2012-2116

[24] J. Wu, T. New, An investigation on supersonic bevelled nozzle jets. Aerospace Science and Technology, 63, 2017: 278-293.

https://doi.org/10.1016/i.ast.2017.01.003

[25] E. Rathakrishnan, Applied gas dynamics. John Wiley \& Sons; 2019 Apr 29.

[26] F. Menter, Zonal two-equation k- $\omega$ turbulence models for aerodynamic flows, 1993: AIAA paper 93-2906.

https://doi.org/10.2514/6.1993-2906

[27] D. Wilcox, Formulation of the $\mathrm{k}-\omega$ turbulence model revisited. AIAA journal, 46 (11), 2008: 2823-2838.

https://doi.org/10.2514/1.36541

[28] S. Hromisin, J. Lampenfield, D. McLaughlin, P. Morris, Experimental and numerical study of injector design and operation on supersonic jet noise reduction using fluidic corrugations. In $22^{\text {nd }}$ AIAA/CEAS Aero acoustics Conference, 2016, p.2989.

https://doi.org/10.2514/6.2016-2989

[29] J. Morgan, P. Morris, D. McLaughlin, C. Prasad, Further development of supersonic jet noise reduction using nozzle fluidic inserts. In $55^{\text {th }}$ AIAA aerospace sciences meeting, 2017, p. 0683.

https://doi.org/10.2514/6.2017-0683

[30] P. Bradshaw, Turbulent secondary flows. An RFM, 19, 1987: 53-74.

https://doi.org/10.1146/annurev.fl.19.010187.000 $\underline{413}$

[31] J. Anderson, Modern compressible flow. Tata McGraw-Hill Education; 2003. 